\title{
CONCERNING PERIODICITY IN THE ASYMPTOTIC BEHAVIOUR OF PARTITION FUNCTIONS
}

\author{
Dedicated to George Szekeres on his 65th birthday \\ P. ERDÖS and B. RICHMOND
}

(Received 31 December 1974)

Communicated by J. Wallis

\begin{abstract}
Let $P_{A}(n)$ denote the number of partitions of $n$ into summands chosen from the set $A=\left\{a_{1}, a_{2}, \cdots\right\}$. De Bruijn has shown that in Mahler's partition problem $\left(a_{\nu}=r^{2}\right)$ there is a periodic component in the asymptotic behaviour of $P_{A}(n)$. We show by example that this may happen for sequences that satisfy $a_{\nu} \sim \nu$ and consider an analogous phenomena for partitions into primes. We then consider corresponding results for partitions into distinct summands. Finally we obtain some weaker results using elementary methods.
\end{abstract}

\section{Introduction}

Let $A=\left\{a_{0}, a_{1}, \cdots\right\}$ be an infinite set of monotone increasing integers. Let $p_{A}(n)$ denote the number of ways of representing $n$ as the sum of summands chosen from $A$. Mahler (1940) showed that when $a_{\nu}=r^{\nu}$ as $n \rightarrow \infty$

$$
\begin{aligned}
\log P_{A}(r n)= & \frac{1}{2 \log r}\left(\log (n(\log n))^{2}+\left(\frac{1}{2}+\frac{1}{\log r}+\frac{\log \log r}{\log r}\right) \log n\right. \\
& -\left(1+\frac{\log \log r}{\log r}\right) \log \log n+0\{1\} .
\end{aligned}
$$

De Bruijn (1943) has shown that this 0-term is actually of the form

$$
U\left(\frac{\log n-\log \log n+\log \log r}{\log r}\right)+0\left\{\frac{(\log \log n)^{2}}{\log n}\right\}
$$

where $U$ is a periodic function with period one and de Bruijn determined the Fourier expansion of $U$. This result has been generalized by various authors, for zxample, Pennington (1953) and Schwarz (1967) and it seems to be common with sets $A$ which have $\lim \inf \left(\log a_{\nu}\right) / \nu>0$ for there to be a periodic or almost 
periodic function analogous to de Bruijn's $U$. In this paper we consider the question of whether partition functions $p_{A}(n)$ with $\lim \inf _{\nu \rightarrow \infty}\left(\log a_{\nu}\right) / \nu=0$ may also exhibit the de Bruijn-Mahler phenomenon. We first show that $p_{A}(n)$ with $A$ defined to be the set of integers which are not positive powers of a fixed integer has a periodic term very similar to that of de Bruijn (see theorem 1). We then investigate how large this term corresponding to de Bruijn's $U$ function may be. It is seen that when $\boldsymbol{A}$ is the set of primes it may be large indeed and we obtain a direct connection with the Riemann hypothesis. In this case there is a close analogy with the number of primes less than a given limit.

Finally we consider these questions for $q_{A}(n)$ the number of partitions into distinct summands chosen from the set $A$ and obtain some results using elementary methods.

\section{2}

Let $A$ be the set of integers which are not powers of the fixed integer $r$. Then the number $p_{A}(n)$ of partitions of $n$ into summands from $A$ satisfied (from the theorem of Roth and Szekeres (1954))

$$
p_{A}(n)=\left(2 \pi A_{2}\right)^{-\frac{1}{2}} \exp \left\{\alpha n-\sum_{a \in A} \log \left(1-e^{-\alpha a}\right)\right\}\left[1+0\left\{n^{-\frac{1}{2}}\right\}\right]
$$

where

$$
A_{2}=\sum_{a \in A} a^{2} \frac{e^{\alpha a}}{\left(e^{\alpha a}-1\right)^{2}}
$$

and $\alpha$ is defined by

$$
n=\sum_{a \in A} \frac{a}{e^{\alpha a}-1} .
$$

From Mellin's transformation formula

$$
\sum_{a \in A} \frac{a}{e^{\alpha a}-1}=\frac{1}{2 \pi i} \int_{\sigma-i x}^{\sigma+i x} \alpha^{-t} \Gamma(t) \zeta(t) \zeta_{A}(t-1) d t \quad(\sigma>2)
$$

where $\Gamma(t)$ denotes the usual gamma-function, $\zeta(t)$ denotes the Riemann zeta-function and

$$
\zeta_{A}(t)=\sum_{a \in A} a^{-t}
$$

Now

$$
\zeta_{A}(t)=\sum_{n=1}^{\infty} n^{-t}-\sum_{i=1}^{\infty} r^{-j t}=\zeta(t)-\frac{1}{r^{t}-1},
$$


thus $\zeta_{A}(t)$ is defined in the entire plane except for simple poles at $t=1$ and $t=2 \pi i k / \log r(k=0,1,2, \cdots)$. The arguments of Pennington (1953) show that we may shift the contour of integration to a line $\sigma<0$ and obtain (using $\left.\zeta(0)=-\frac{1}{2}\right)$ that

$$
\begin{aligned}
n= & \alpha^{-2} \pi^{2} / 6-\alpha^{-1} \frac{\log (1 / \alpha)}{\log r} \\
& -\frac{1}{\alpha \log r} \sum_{\nu}^{\prime} \Gamma\left(1+\frac{2 \pi i \nu}{\log r}\right) \zeta\left(1+\frac{2 \pi i \nu}{\log r}\right) \exp \left(-\frac{2 \pi i \nu \log \alpha}{\log r}\right) \\
& +0\{1\}
\end{aligned}
$$

where $\gamma$ denotes Euler's constant and $\Sigma_{\nu}^{\prime}$ denotes summation over nonzero $\nu$.

We may solve this for $\alpha$ to obtain

$$
\begin{aligned}
\alpha= & n^{-1 / 2} \frac{\pi}{\sqrt{6}}\left[1-n^{-1 / 2} \frac{\sqrt{6}}{2 \pi \log r}\left\{\log \frac{\sqrt{6}}{\pi}+\right.\right. \\
& \left.\sum_{\nu} \Gamma\left(1+\frac{2 \pi i \nu}{\log r}\right) \zeta\left(1+\frac{2 \pi i \nu}{\log r}\right) \exp \left(2 \pi i \nu \log \left(\frac{\sqrt{6 n}}{\pi}\right)\right\}\right] \\
& +0\left\{n^{-2 / 3} \log ^{2} n\right\} .
\end{aligned}
$$

From Mellin's transformation formula

$$
-\sum_{a \in A} \log \left(1-e^{-\alpha a}\right)=\frac{1}{2 \pi i} \int_{\sigma-i \alpha}^{\sigma+i \alpha} \alpha^{-t} \zeta(t+1) \Gamma(t) \zeta_{A}(t) a t \quad(\sigma>1) .
$$

Again shifting the contour of integration to $\sigma<0$, using $\zeta^{\prime}(0)=\frac{1}{2} \log 2 \pi$ and that (with $\left.z \zeta(z+1)=1+\gamma z+\gamma_{2} z^{2}+\cdots\right)$

$$
\zeta(t+1) \Gamma(t) \zeta_{A}(t)=\frac{-1}{t^{3} \log r}-\left\{\frac{\log ^{2} r}{12}-\frac{1}{2} \log 2 \pi+\frac{\gamma_{2}+\frac{\gamma^{2}}{2}+\frac{\pi^{2}}{12}}{\log r}\right\} \frac{1}{t}+0\{1\}
$$

yields

$$
\begin{aligned}
-\sum_{a \in A} \log \left(1-e^{-\alpha a}\right)= & \alpha^{-1} \frac{\pi^{2}}{6}-\frac{\log ^{2}(1 / \alpha)}{\log r}+\frac{\log r}{12}+\frac{1}{2} \log 2 \pi \\
& -\frac{\gamma_{2}+\frac{\gamma^{2}}{2}+\frac{\pi^{2}}{12}}{\log r}-\frac{1}{\log r} \sum_{\nu}^{\prime} \zeta\left(1+\frac{2 \pi i \nu}{\log r}\right) \Gamma\left(\frac{2 \pi i \nu}{\log r}\right) \\
& \times \exp \left(\frac{2 \pi i \nu \log \alpha}{\log r}\right)+0\{\alpha\}
\end{aligned}
$$


From (2.4) and (2.6) we obtain

$$
\begin{aligned}
\alpha n-\sum_{\alpha \in A} \log \left\{1-e^{-\alpha a}\right\}= & \alpha^{-1} \frac{\pi^{2}}{3}-\frac{\log ^{2}(1 / \alpha)}{\log r}-\frac{\log (1 / \alpha)}{\log r}+\frac{1}{2} \log 2 \pi \\
& -\frac{\log r}{12}-\frac{\gamma_{2}+\frac{\gamma^{2}}{2}+\frac{\pi^{2}}{12}}{\log r}-\frac{1}{\log r} \sum_{\nu}^{\prime} \zeta\left(1+\frac{2 \pi i \nu}{\log r}\right) \\
& \times\left(1+\frac{2 \pi i \nu}{\log r}\right) \Gamma\left(\frac{2 \pi i \nu}{\log r}\right) \exp \left(\frac{2 \pi i \nu \log \left(\alpha^{-1}\right)}{\log r}\right) \\
& +0\{\alpha\} .
\end{aligned}
$$

Note that

$$
A_{2}=\frac{d}{\alpha a}\left[\sum_{a} \frac{a}{e^{\alpha a}-1}\right]=\frac{\alpha^{-3} \pi^{2}}{6}+0\left\{\alpha^{-2}\right\} \text {. }
$$

Thus from (2.7), (2.8) and (2.5) applied to (2.1) we obtain

THEOREM 1. Let $A$ be the set of integers which are not positive powers of the fixed integer $r$. Then

$$
\begin{aligned}
\log p_{A}(n)= & \pi n^{\frac{1}{2}} \sqrt{\frac{2}{3}}-\frac{\log ^{2}(\sqrt{6 n} / \pi)}{\log r}+\frac{3}{\log \left(\frac{\sqrt{6 n}}{\pi}\right)} \\
& -\frac{\log r}{12}-\frac{1}{2} \log \left(\frac{\pi^{2}}{3}\right)-\frac{\gamma_{2}+\gamma+\frac{\gamma^{2}}{2}+\frac{\pi^{2}}{12}}{\log r}-\frac{1}{\log r} \\
& \times \sum_{\nu}^{\prime} \zeta\left(1+\frac{2 \pi \nu}{\log r}\right) \Gamma\left(\frac{2 \pi i \nu}{\log r}\right) \exp \left(\frac{2 \pi i \nu}{\log r} \log \left(\frac{\sqrt{6 n}}{\pi}\right)\right) \\
& +0\left\{n^{-1 / 2} \log ^{2} n\right\} .
\end{aligned}
$$

This theorem shows that even sequences for which $a_{\nu} / \nu=1+0\left\{\nu^{-1} \log \nu\right\}$ may exhibit the de Bruijn-Mahler phenomenon. The asymptotic formula is in terms of elementary functions. In our attempt to determine how large this oscillatory component may be we shall consider an example in which the asymptotic formula cannot be so expressed.

THEOREM 2. Let $A$ be the set of primes. Let $\alpha=\alpha(n)$ be defined by

$$
n=\sum_{a \in A} \frac{a}{e^{\alpha a}-1}
$$

then 


$$
\alpha \sim \frac{\pi}{\sqrt{3}} n^{-1 / 2} \log ^{-1 / 2} n
$$

Let

$$
F(\alpha)=\alpha^{-1} \int_{0}^{\infty} e^{-u \log (1 / \alpha)}(2+u) \Gamma(1+u) \zeta(2+u) d u
$$

Thus by standard results on the Laplace Transform

$$
F(\alpha)=\alpha^{-1} \sum_{r=0}^{N} \frac{C_{n} n !}{\log ^{n+1} \frac{1}{\alpha}}+0\left\{\alpha^{-1} \log ^{-N+2} \frac{1}{\alpha}\right\}
$$

where

$$
(2+u) \Gamma(1+u) \zeta(2+u)=\sum_{n=0}^{\infty} C_{n} u^{n}, \quad C_{0}=\frac{\pi^{2}}{3}
$$

a) There exists a constant $C>0$ such that

$$
\log P_{A}(n)=F(\alpha)+0\left\{\alpha^{-1} \exp \left(-C \log ^{\frac{3}{5}}\left(\frac{1}{\alpha}\right) \log \log ^{-\frac{1}{5}} \frac{1}{\alpha}\right)\right\} \text {. }
$$

b) Let $\theta \equiv$ l.u.b. of the real parts of the imaginary roots of the Riemann zeta-function. Then for every $\varepsilon>0$

$$
\log p_{A}(n)-F(\alpha)=0\left\{\alpha^{-\theta+\varepsilon}\right\} .
$$

c) Conversely if

$$
\log p_{\mathrm{A}}(n)-F(\alpha)=0\left\{\alpha^{-\psi}\right\}
$$

then $\theta \leqq \psi$ where $\theta$ is defined in part b).

Proof. The proof of parts a) and b) is very similar to classical proofs in the :heory of primes. It is also very similar to the proof of Theorem 7.1 of Richmond. In particular it is shown in Richmond that

$$
\alpha \sim \frac{\pi}{\sqrt{3}} n^{-\frac{1}{2}} \log ^{-\frac{1}{2}} n .
$$

From the Roth-Szekeres (1954) theorem and the Mellin transformation formula it follows that

$$
\log p_{A}(n)=\frac{1}{2 \pi i} \int_{\sigma-i \infty}^{\sigma+i x} \alpha^{-t}(1+t) \Gamma(t) \zeta(t+1) \zeta_{A}(t) d t+0\{\log n\}, \quad(\sigma>1)
$$

where

$$
\zeta_{A}(t)=\sum_{a \in A} a^{-t}
$$


It is well-known that (see p. 12 of Tichmarsh (1951))

$$
\zeta_{A}(t)=\log \zeta(t)+h(t), \quad h(t)=\sum_{m=2}^{\infty} \frac{\zeta_{A}(m t)}{m}
$$

where $h(t)$ is holomorphic for $R t>\frac{1}{2}$. One obtains with standard residue arguments that

$$
\begin{gathered}
\frac{1}{2 \pi i} \int_{\sigma-i \infty}^{\sigma+i \infty} \alpha^{-t}(1+t) \Gamma(t) \zeta(t+1) \log \frac{1}{t-1} d t \\
=F(\alpha)+0\left\{\log \frac{1}{\alpha}\right\} .
\end{gathered}
$$

There is a constant $C>0$ such that $\zeta(t)$ has no zeros in (p. 87 of Montgomery (1971))

$$
R t=1-\frac{C}{\log ^{2 / 3}|t| \log \log { }^{1 / 3}|t|}
$$

Thus by a classical argument (pp. 77-88 of Prachar (1957) by the $\Gamma$-function or see Richmond)

$$
\begin{aligned}
\frac{1}{2 \pi i} \int_{\sigma-i \infty}^{\sigma+i \infty} \alpha^{-t}(1+t) \Gamma(t) \zeta(t+1)\left[\zeta_{A}(t)\right. & \left.-\log \frac{1}{t-1}\right] d t \\
& =0\left\{\alpha^{-1} e^{-c} \log ^{\frac{3}{3}}\left(\frac{1}{\alpha}\right) \log \log ^{-1 / 5}\left(\frac{1}{\alpha}\right)\right\} .
\end{aligned}
$$

This proves part a) of the theorem and part b) follows in the same way.

To prove part c) note that

$$
\begin{aligned}
\log p_{A}(n)-F(\alpha)= & \frac{1}{2 \pi i} \int_{\sigma-i \infty}^{\sigma+i \infty} \alpha^{-t}(1+t) \Gamma(t) \zeta(1+t)\left(\zeta_{A}(t)-\log \frac{1}{t-1}\right) d t \\
& +0\left\{\log \frac{1}{\alpha}\right\} .
\end{aligned}
$$

We can think of $p_{A}(n)$ as a function of $\alpha$ and it is known that (see Roth and Szekeres (1954) or Richmond)

$$
\begin{aligned}
\alpha(n+1)-\alpha(n) & =0\left\{\alpha^{2} \log \frac{1}{\alpha}\right\} \\
\log p_{A}(n+1)-\log p_{A}(n) & =0\left\{\alpha \log \frac{1}{\alpha}\right\} .
\end{aligned}
$$

From this one may readily deduce (Richmond) that $F(\alpha(n+1))-F(\alpha(n))=$ $0\{\alpha \log 1 / \alpha\}$ hence we suppose the hypothesis of c) to hold for all $\alpha$.

By the Mellin inversion formula 


$$
(1+t) \zeta(1+t) \Gamma(t)\left\{\zeta_{A}(t)-\log \frac{1}{t-1}\right\}=\int_{0}^{\infty} \alpha^{t-1}\left[p_{A}(n(\alpha))-F(\alpha)\right] d \alpha .
$$

The integral on the right converges for all $t$ with $R t>\psi$, hence represents a holomorphic function for $R t>\psi$. By eq. (2.9) we obtain our result.

The proof of part c) of Theorem 2 shows that one cannot have

$$
\log p_{A}(n)-F(\alpha)=0\left\{\alpha^{\frac{1}{2}-\theta}\right\}
$$

for any positive constant $\theta$ since $\zeta_{A}(t)-\log (1 /(t-1)$ is not bounded at $t=1 / 2$. Also parts b) and c) show that the size of $\log P_{A}(n)-F(\alpha)$ is directly related to the question of where the roots of $\zeta(t)$ lie.

To see that $\log P_{A}(n)-F(\alpha)$ corresponds to the de Bruijn-Mahler phenomenon we consider the following representation:

Let $\rho=\beta+i \gamma$ run through the complex zeros of $\zeta(t)$. Bracket all zeros such that any two zeros for which

$$
\left|\gamma-\gamma^{\prime}\right|<\exp (-A \gamma / \log \gamma)+\exp \left(-A \gamma^{\prime} / \log \gamma^{\prime}\right)
$$

are included in the same bracket. With the bracketing above by the arguments of Tichmarsh (1951) (on pp. 185-187)

$$
\log p_{A}(n)-F(\alpha)=\sum_{\rho} \operatorname{Res}\left\{\alpha^{-s}(1+S) \Gamma(S) \zeta(S+1) \zeta_{A}(S)\right\}_{S=\rho}+0\left\{\log \frac{1}{\alpha}\right\} .
$$

\section{3}

In this section we consider the corresponding problems for $q_{A}(n)$, the number of partitions of $n$ into distinct summands chosen from $A$. The Roth $\uparrow$ Szekeres results and the Mellin transformation techniques in $\$ 2$ apply with minor modifications and we have

THEOREM 3. Let $A$ be the set of integers which are not positive powers of a fixed integer $r$. Then

$$
\begin{aligned}
\log q_{A}(n)= & \frac{\pi}{\sqrt{3}} n^{1 / 2}-\frac{\log 2}{\log r} \log \left(\frac{\sqrt{12 n}}{\pi}\right)-\frac{3}{4} \log n+\frac{\log ^{2} s}{2 \log r}-\frac{1}{4} \log (48) \\
& +\frac{\log 2}{2}-\frac{1}{\log r} \sum_{\nu}^{\prime}\left(1-2^{-2 \pi i \nu / \log }\right) \\
& \times \zeta\left(1+\frac{2 \pi i \nu}{\log r}\right) \exp \left(\frac{2 \pi i \nu}{\log r} \log \left(\frac{\sqrt{12 n}}{\pi}\right)\right) \Gamma\left(\frac{2 \pi i \nu}{\log r}\right)+0\left\{n^{-1 / 2} \log ^{2} n\right\} .
\end{aligned}
$$

Note that when $r=2$ the oscillatory component drops out and the next theorem shows that this must be expected. 
THEOREM 4. Let $A$ be the set of integers which are not positive powers of 2. Let $q(n)$ denote the number of partitions of $n$ into distinct integers. Then

$$
q_{A}(n)=q(n)-q(n-2) .
$$

Proof. This theorem follows from generating series techniques however we give the following proof due to H. Shank. We wish to prove that $q(n-2)$ is the number of partitions of $n$ into distinct summands at least one of which is a power of 2 . Let $2^{i}$ be the smallest power of 2 in such a partition of $n$. Then $2^{i}-2=2+2^{2}+\cdots+2^{i-1}$ and we obtain a partition of $n-2$ into distinct summands. Clearly different partitions of $n$ give different partitions of $n-2$. On the other hand suppose we have a partition of $n-2$ into distinct parts. If it contains a 2 let $2,2^{2}, \cdots, 2^{i}$ be the longest string of consecutive powers of 2 it contains. We may replace this string of $2^{i}$ 's by $2^{i+1}$ to obtain a partition of $n$. In this way different partitions of $n-2$ give rise to different partitions of $n$ containing a positive power of 2 . We now prove

TheOREM 5. Let $A$ be the set of integers which are not positive powers of a fixed integer $r$. Let $k$ be any constant integer. Then for all sufficiently large $n$ the $k$-th differences of $p_{A}(n)$ and $q_{A}(n)$ are positive.

Proof. The result for $p_{A}(n)$ follows at once from the work of Bateman and Erdös (1956) since if one removes an arbitrary subset of $A$ having elements, the remaining elements of $A$ have greatest common divisor unity.

To prove the result for $q_{A}(n)$ we first of all note that it is sufficient to show that $\Sigma q_{A}(n) x^{n}$ can be written as

$$
\sum q_{A}(n) x^{n}=\sum b_{n} x^{n} \cdot \sum C_{n} x^{n}
$$

where the $k$-th difference of the $b_{n}$ is positive for $n$ sufficiently large and where $C_{n} \geqq 0$. Suppose $r$ has an odd factor $d$. We write

$$
\begin{aligned}
\sum q_{A}(n) x^{n}= & \prod_{i=1}^{\infty}\left(1-x^{i}\right) \cdot \prod_{i=1}^{\infty}\left(1+x r^{i}\right)^{-1} \\
= & \prod_{i=0}^{\infty}\left(1-x^{2 i+1}\right)^{-1} \cdot \prod_{i=1}^{\infty}\left(1+x^{2 r^{i}}\right)^{-1} \cdot \prod_{i=1}^{\infty}\left(1-x^{i}\right) \\
= & \prod_{\substack{j=1(\bmod 2) \\
j \neq d^{i}}}^{\infty} \cdot\left(1-x^{j}\right)^{-1} \\
& \times \prod_{i=1}^{\infty}\left(1+x^{d^{i}}+\cdots+x^{d^{i}}(r / d)^{i} \cdot \prod\left(1-x^{2 r^{i}}\right)^{-1}\right.
\end{aligned}
$$


However the $k$-difference of the coefficients of the first product are positive from some point on by the results of Bateman and Erdös (1956) and we obtain our result from the remark above. Suppose $r=2^{\mathrm{s}}$. Then

$$
\sum q_{A}(n) x^{r}=\prod_{j=1}^{\infty}\left(1-x^{2 j+1}\right)^{-1} \cdot \frac{1-x^{r}}{1-x} \prod_{i=1}^{\infty} \frac{1-x^{r^{i+1}}}{1-x^{2 r^{i}}}
$$

Clearly $2 r^{i} / r^{i+1}$ and we again have that the $k$-difference of the coefficients of the first product are positive from some point on by the results of Bateman and Erdös (1956).

Finally we show by elementary arguments

THEOREM 6. Let $A$ be the set of integers which are not positive powers of a fixed integer $r$.

$$
\begin{aligned}
& \log p_{A}(n)=\sqrt{\frac{2}{2}} \pi n^{\frac{1}{2}}+0\left\{\log ^{2} n\right\} \\
& \log q_{A}(n)=\frac{\pi}{\sqrt{3}} n^{\frac{1}{2}}+0\{\log n\} .
\end{aligned}
$$

Proof. We first prove

$$
q(n)>q_{A}(n)>n^{-1} q(n) .
$$

The first inequality is obvious. Also

$$
\prod_{i=1}^{\infty}\left(1+x^{r^{i}}\right) \cdot \sum q_{A}(n) x^{n}=\sum q(n) x^{n} .
$$

Since the coefficients of the Taylor series expansion of the infinite product are zero or one and since the $q_{A}(n)$ are monotone increasing we readily obtain the second inequality.

Note that

$$
\prod_{i=1}^{\infty}\left(1-x^{r^{\prime}}\right)^{-1} \cdot \sum p_{A}(n) x^{n}=\sum p(n) x^{n} .
$$

The infinite product is $\sum p_{r}(n) x^{n}$ where $p_{r}(n)$ is the number of partitions of $n$ into powers of $r$. Erdös (1942) has shown that $\log p_{r}(n) \sim \log ^{2} n / 2 \log r$. From this and the fact that $p_{A}(n)$ and $p_{r}(n)$ are monotone increasing we obtain that $p(n)>p(n) \exp -\left(\varepsilon+(2 \log r)^{-1}\right) \log ^{2} n$. Erdös (1942) has shown using elementary arguments that $p(n) \sim C n^{-1} \exp \left(n^{\frac{1}{2}} \pi \sqrt{2 / 3}\right)$ (Newman (1951) showed also by elementary arguments that $C=1 /(4 \sqrt{3})$ ). The first part of theorem 6 follows immediately. Since the number of partitions of $n$ into distinct summands equals the number of partitions of $n$ into odd summands one can apply the method of Erdös to obtain $q(n) \sim C n^{-\frac{3}{4}} \exp \left(n^{\frac{1}{2}} \pi / \sqrt{3}\right)$ and this with (3.1) gives the second part of Theorem 6 . 


\section{References}

K. Mahler (1940), 'On a Special Functional Equation', J. London Math. Soc. 15, 115-123.

N. G. de Bruijn (1943), 'On Mahler's Partition Problem', Indag. Math. 10, 210-220.

W. B. Pennington (1953), 'On Mahler's Partition Problem', Annals of Math. (2) 57, 531-546.

W. Schwarz.(1967), 'C. Mahler's Partitions Problem', J. reine angew. Math 229, 182-188.

K. F. Roth and G. Szekeres (1954), 'Some Asymptotic formulae in the Theory of Partitions', Quart. J. Math., Oxford (2), 244-259.

L. B. Richmond, 'Moments of Partitions II', Acta Arith. (to appear).

E. C. Tichmarsh (1951), The Theory of the Riemann Zeta-function (Oxford, 1951).

H. L. Montgomery (1971), Topics in Multiplicative Number Theory (Springer-Verlag, 1971).

K. Prachar (1957), Primzahlverteilung (Springer-Verlag, 1957).

P. T. Bateman and P. Erdös (1956), 'Monotonicity of Partition Functions', Mathematica 3, 1-14.

P. Erdös (1943), 'On an Elementary Proof of Some Asymptotic Formulas in the Theory of Partitions', Annals of Math. (2) 43, 437-450.

D. J. Newman (1951), 'The Evaluation of the Constant in the Formula for the Number of Partitions of $n$ ', Amer. J. of Math., 73, 599-601.

University of Waterloo

University Avenue

Waterloo

Ontario, Canada 